\title{
ON THE CHARACTERS OF AFFINE KAC-MOODY GROUPS
}

\author{
by STEPHEN SLEBARSKI
}

(Received 18th October 1990, revised 17th March 1992)

\begin{abstract}
Let $G$ be an affine Kac-Moody group over $\mathbb{C}$, and $V^{\omega}$ an integrable simple quotient of a Verma module for $\mathrm{g}$. Let $G^{\mathrm{min}}$ be the subgroup of $G$ generated by the maximal algebraic torus T, and the real root subgroups.

It is shown that $\delta \in \Phi_{+}^{\text {im }}$ (the least positive imaginary root) gives a character $\delta \in \operatorname{Hom}\left(G, \mathbb{C}^{*}\right)$ such that the pointwise character $\chi^{\infty}$ of $V^{\omega}$ may be defined on $G^{\min } \cap G^{>1}$.
\end{abstract}

1980 Mathematics subject classification: 17B67, 22E67.

\section{Introduction}

A Kac-Moody group $G$ over $\mathbb{C}$, is associated to a pair $\left(A, \mathfrak{h}_{\mathbf{z}}\right)$ where $A$ is a generalized, indecomposable, Cartan $n \times n$ matrix of rank $l$, and $\mathfrak{h}_{\mathbf{z}}$ is a free $\mathbb{Z}$-module such that $n-l=\operatorname{rank} \mathfrak{h}_{z}-n$. Then $G$ has a $(B, N)$ pair forming a Tits system with Weyl group $W=N /(B \cap N)$ (see also $[12,4,9]$ ).

The Lie algebra $g$ of $G$ has a root space decomposition, and it is required that the roots $\Phi \subseteq \operatorname{Hom}\left(\mathfrak{h}_{\mathbf{z}}, \mathbb{Z}\right)=: \mathfrak{h}_{\mathbf{Z}}^{*}$. We have $\Phi=\Phi^{\text {re }} \cup \Phi^{\text {im }}$, where $\Phi^{\text {re }}$ is the $W$ orbit of the simple roots and $\Phi^{\mathrm{im}}=\Phi \backslash \Phi^{\mathrm{re}}$.

If $G$ is affine (that is $A$ is symmetrizable, positive semidefinite) then there is an analytic construction as a loop group [3]. Take a central extension $S^{1} \rightarrow \tilde{L} K_{(0)} \rightarrow L K_{(0)}$ of the loop group of a compact, connected almost simple Lie group $K_{(0)}$ by the circle $S^{1}$ (this is obtained [8] from a closed, left invariant integral 2 -form on $L K_{(0)}$, if $K_{0}$ is simply connected). Imbed $K_{(0)}$ in a group of finite dimensional unitary matrices, and let $L_{\text {pol }} K_{(0)}$ be the dense subgroup of $L K_{(0)}$ consisting of $\gamma: S^{1} \rightarrow K_{(0)}$ with each matrix entry of $\gamma(z)$ a finite Laurent polynomial in $z$. The loop algebra $L \mathfrak{f}_{(0)}=\mathbb{C}\left[z, z^{-1}\right] \otimes_{\mathbf{R}} \mathfrak{f}_{(0)}$ has a derivation $d$ by $z \frac{d}{d z} \otimes 1$, and on $\tilde{L} \mathfrak{f}_{(0)}, d(c)=0$. In 3 the untwisted affine Lie algebra is $\mathfrak{g}=\mathbb{C} d \oplus \mathbb{C} c \oplus L \mathfrak{f}_{(0) \mathcal{c}}$. There is a subgroup $S^{1}$ of the group of diffeomorphisms of the circle, having Lie algebra $\mathbb{R} d$ as a subalgebra of the Virasoro algebra. Set $G_{1}=$ $\left(S^{1} \ltimes \tilde{L}_{\mathrm{pol}} K_{(0)}\right)_{\mathrm{c}}$. The Lie algebra $g$ decomposes (restricting the adjoint representation of $G_{1}$ ) by characters of $S^{1} \otimes T_{(0)}$, where $T_{(0)}$ is a maximal torus of $K_{(0)}$. The Weyl group $W=W_{0} \ltimes \Upsilon$ where $W_{0}$ is the Weyl group of $\left(K_{(0)}, T_{(0)}\right)$ and $\Upsilon$ is the cocharacter lattice $\operatorname{Hom}\left(S^{1}, T_{(0)}\right)$. The "twisted" loop groups are obtained by the outer automorphisms of $\mathfrak{f}_{(0) \mathrm{c}}$ of orders 2,3 .

An algebraic construction (as in $[6,9]$ ) for general $G$ is used here. This is obtained as a subgroup of $\mathrm{GL}(V)$ where $V$ is the direct sum of the "integrable" simple quotients $V^{\omega}$ 
of Verma modules for g. And see [12] for the Chevalley-Demazure, and Tits group functor on the category of rings.

To briefly describe a correspondence between the analytic approach and the algebraic of (1.3), (3.1):

Let $B_{1}^{-}$be the group of polynomial maps $\gamma:\{z \in \mathbb{C} ;|z| \leqq 1\} \rightarrow K_{(0) c}$ restricted to $S^{1}$, with $\gamma(0) \in B_{(0)}^{-}$where $B_{(0)}^{-}$is the opposite Borel subgroup to $B_{(0)} \leqq K_{(0) C}$, the latter associated to a choice of positive roots $\Phi_{0+}$ for $\left(K_{(0)}, T_{(0)}\right)$. Let $U_{(0) \alpha}$ be the root subgroup in $K_{(0) c}$ of $\alpha \in \Phi_{0+}$, and define $U_{\alpha_{i}}=\left\{\gamma_{g} \in B_{1} ; \operatorname{Im} \gamma_{g}=\{g\}, g \in U_{(0) \alpha_{s}}\right\}, i \neq 0$, $U_{a_{0}}=\left\{\gamma \in B_{1} ; \gamma^{(1)}(0) \in U_{(0)-\theta}, \gamma^{(s)}(0)=0, s \neq 1\right\}, \theta \in \Phi_{0+}$ the highest root. Let $B^{-}=S^{1} \times \widetilde{B}_{1}^{-}$. Over a completion of $G_{1} / B^{-}$there is a holomorphic $G_{1}$ vector bundle $G_{1} \times{ }_{B}-\mathbb{C}_{\omega}, \omega$ a character of $B^{-}$which is trivial on $U^{-}$. The Borel-Weil theorem for compact Lie groups has a generalization to loop groups (see for example [8]). In particular the $G_{1}$-space of holomorphic sections $H^{0}(\omega)$ is $\mathrm{g}$ equivalent to $V^{\omega}$. The group $G$ in Section 3 is the homomorphic image in $G L(V)$ of $G_{1}$ (and see [8, p. 144] for the Bruhat decomposition of $G_{1}$ ).

In this paper, for $G$ affine, we give the subdomain of $G^{\text {min }}$ on which a pointwise character $\chi^{\omega}$ of the representation $\left(V^{\omega}, R\right), \omega \in \mathscr{I} \mathrm{nt}_{+} \cap \mathfrak{h}_{Z}^{*}$ can be defined. Here $G^{\text {min }}$ is the subgroup of $G$ generated by the algebraic torus $T=h_{\mathbf{Z}} \otimes_{\mathbf{Z}} \mathbb{C}^{*}$ and the root subgroups $U_{\alpha}, \alpha \in \Phi^{\text {re }}$. We show that this domain is given by $G^{>1}=\{g \in G ;|\delta(g)|>1\}$ where $\delta \in \Phi_{+}^{\mathrm{im}}$ is the least positive imaginary root trivially extended to $\delta \in \operatorname{Hom}\left(G, \mathbb{C}^{*}\right)$. The proof holds for twisted $G$, and the present approach does not exploit the topology as a loop group. The subdomain in $T$ on which $\chi^{\omega}$ behaves well analytically is known in general ([4], and also [10] for $N$ the normalizer of $T$ in $G$ ). Then to prove that $G^{>1}$ is the set of elements of $G$ acting as Hilbert-Schmidt operators on $V^{\omega}$, we use that (1) $V^{\omega}$ is a pre-Hilbert space with $K$ acting as unitary operators (2) the complex Iwasawa decomposition $G=K B$, and (3) a Levi subgroup $L_{1}$ of $G$ of finite type has a $K_{1} T K_{1}$ decomposition, $K_{1}=L_{1} \cap K$. These elements $g \in G^{>1}$ have a trace which is denoted $\chi^{\omega}(g)$, and $\chi^{\omega}$ is shown to be $G$-conjugation invariant there. A corollary to this result is an affirmative answer to the remark in $[8, p .275]$.

\section{Notation and preliminary results}

1.1. Let $G$ be a Kac-Moody group associated to the root datum $\left(h_{z}, \Delta^{v}, \Delta\right)$. That is (see also (1.2). (1.3)) from a general Cartan $n \times n$ matrix $A$ of rank $l$ we take a free $\mathbb{Z}$ module $\mathfrak{h}_{\mathbf{z}}$ of finite rank and $\mathbb{Z}$ independent subsets $\Delta^{v}=\left\{h_{1}, \ldots, h_{n}\right\} \subseteq \mathfrak{h}_{\mathbf{Z}}$ "the simple coroots", $\Delta=\left\{\alpha_{1}, \ldots, \alpha_{n}\right\} \subseteq \mathfrak{h}_{\mathbf{Z}}^{*}=\operatorname{Hom}\left(\mathfrak{h}_{\mathbf{Z}}, \mathbb{Z}\right)$ "the simple roots" with $\alpha_{j}\left(h_{i}\right)=a_{i j}, \forall i, j$ and $n-l=\operatorname{rank} \mathfrak{h}_{\mathbf{z}}-n$.

The Weyl group $W$ of $\left(h_{z}, \Delta^{v}, \Delta\right)$ is a Coxeter group generated by reflections $r_{i}: \mathfrak{h}_{\mathbf{Z}} \rightarrow \mathfrak{h}_{\mathbf{Z}}, r_{i}(h)=h-\alpha_{i}(h) h_{i}, h \in \mathfrak{h}_{\mathbf{z}}$ and acts (faithfully) contragrediently on $\mathfrak{h}_{\mathbf{Z}}^{*}$.

There is a Lie algebra $\mathfrak{g}=\mathrm{g}(A)$ with bracket [] and adjoint representation ad, generated by $\mathfrak{h}=\mathfrak{h}_{\mathbf{Z}} \otimes_{\mathbf{z}} \mathbb{C}, e_{i}, f_{i}, i=1, \ldots, n$ with relations $\left[h, h^{\prime}\right]=0, \quad\left[h, e_{i}\right]=\alpha_{i}(h) e_{i}$, $\left[h, f_{i}\right]=-\alpha_{i}(h) f_{i}, \quad\left[e_{i}, f_{j}\right]=\delta_{i j} h_{j}, \quad\left(\operatorname{ad} e_{i}\right)^{-a_{i j}+1}\left(e_{j}\right)=0, \quad\left(\operatorname{ad~} f_{i}\right)^{-a_{i j}+1}\left(f_{j}\right)=0, \quad \forall h, h^{\prime} \in \mathfrak{h}, i$, 
$j \in\{1, \ldots, n\}$. Also by taking the factor Lie algebra, we may assume that the $\mathfrak{h}$ radical of $\mathfrak{g}$ is zero; that is every ideal of $\mathfrak{g}$ which intersects $\mathfrak{h}$ trivially is zero.

Then $\mathfrak{g}$ is $\mathbb{Z} \Delta$-graded and has a triangular decomposition $\mathfrak{g}=\mathbf{n}_{-} \oplus \mathfrak{h} \oplus \mathbf{n}_{+}$over $\mathbb{C}$. If $A$ is indecomposable, then $\mathrm{g}$ is simple if and only if $\operatorname{det} A \neq 0$. The root space decomposition is $\mathfrak{g}=\sum_{\alpha \in \mathfrak{h}^{*}}^{\oplus} \mathfrak{g}_{\alpha}$ where $\mathfrak{g}_{\alpha}=\{x \in \mathfrak{g} ;[h x]=\alpha(h) x, \forall h \in \mathfrak{h}\}$ with roots $\Phi=$ $\left\{\alpha \in \mathfrak{h}^{*} ; \mathfrak{g}_{\alpha} \neq 0\right\}$. The Cartan subalgebra $\mathfrak{h}=\mathfrak{g}_{0}$. We have $\mathfrak{g}_{\alpha_{i}}=\mathbb{C} e_{i}, \mathfrak{g}_{-a_{i}}=\mathbb{C} f_{i}$ and $\mathfrak{n}_{ \pm}=$ $\sum_{\alpha \in \Phi_{+}}^{\oplus} g_{ \pm \alpha}$ where $\Phi_{+}=\Phi \cap \mathbb{N} \Delta, \Phi_{-}=-\Phi_{+}$.

The root system $\Phi$ is invariant under $W$. The multiplicity of the root $\alpha$, mult $\alpha$ is $\operatorname{dim}$ $\mathfrak{g}_{\alpha}=\operatorname{dim} \mathfrak{g}_{w(\alpha)}, w \in W$. Let $\Phi^{\text {re }}=W . \Delta$ the real roots, $\Phi^{\text {im }}=\Phi \backslash \Phi^{\text {re }}$ the imaginary roots. Then mult $\alpha=1, \forall \alpha \in \Phi^{\text {re }}$. The set of positive imaginary roots $\Phi_{+}^{\mathrm{im}}$ is $W$-invariant.

If $A$ is symmetrizable (see also (2.1)) then $\mathrm{g}$ carries a symmetric nondegenerate $\mathbb{C}$ bilinear form (,), which is infinitesimally invariant under the adjoint representation ad. This restricts to a nondegenerate form on $\mathfrak{h}$, and gives an isomorphism $v: \mathfrak{h} \rightarrow \mathfrak{h}^{*}, v(h)\left(h^{\prime}\right)=\left(h, h^{\prime}\right), \forall h, h^{\prime} \in \mathfrak{h}$.

1.2. The universal enveloping algebra $u(\mathfrak{g})$ is $\mathbb{Z} \Delta$-graded. Let $\mathfrak{b}=\mathfrak{h} \oplus \mathfrak{n}_{+}$, a standard Borel subalgebra. The line $\mathbb{C}_{\omega}, \omega \in \mathfrak{h}^{*}$ is a $u(\mathfrak{b})$-module by $x .1=0, x \in \mathfrak{n}_{+}, h .1=$ $\omega(h) 1, h \in \mathfrak{h}$. Then define the Verma module $M^{\omega}=u(\mathfrak{g}) \otimes_{u(b)} \mathbb{C}_{\omega}$ with $u(g)$ acting on the left. If $M^{\prime}$ is the maximal $g$-submodule not containing $1 \otimes \mathbb{C}_{\omega}$, then $V^{\omega}=M^{\omega} / M^{\prime}$ is simple. In particular $V^{\omega}=\sum_{\lambda \in h^{*}}^{\oplus} V_{\lambda}$ an h-diagonalization into finite dimensional weight spaces. Denote the set of weights by $P^{\omega}:=P\left(V^{\omega}\right)$. This is partially ordered by the natural filtration of $u(\mathfrak{g})$, with the highest weight $\omega$ minimal. If $\alpha=\sum_{i} c_{i} \alpha_{i} \in \mathbb{N} \Delta$, the height $h t(\alpha)=\sum_{i} c_{i}$. The support $\operatorname{supp}(\alpha)=\left\{i ; c_{i} \neq 0\right\}$ is connected as a subdiagram of the Coxeter-Dynkin diagram of $W$, if $\alpha \in \Phi_{+}$. And if $\lambda=\omega-\sum_{i} c_{i} \alpha_{i} \in \omega-\mathbb{N} \Delta$, the depth $\operatorname{dep}(\lambda):=\sum_{i} c_{i}$.

Define for root datum $\left(\mathfrak{h}_{\mathbf{Z}}, \Delta^{\vee}, \Delta\right), \mathscr{I} \mathrm{nt}=\left\{\lambda \in \mathfrak{h}^{*} ; \lambda\left(h_{i}\right) \in \mathbb{Z}, i=1, \ldots, n\right\}$ "the lattice of integral forms", $\mathscr{I} \mathrm{nt}_{+}=\left\{\lambda \in \mathfrak{h}^{*} ; \lambda\left(h_{i}\right) \in \mathbb{N}, i=1, \ldots, n\right\}$ "the dominant integral forms", $\mathscr{I} \mathrm{nt}_{++}=\left\{\lambda \in \mathscr{I} \mathrm{nt}_{+} ; \lambda\left(h_{i}\right) \neq 0, i=1, \ldots, n\right\}$ "the strictly dominant forms". Therefore $\Phi \subseteq$ Int. The "fundamental weights" are $\left\{\omega_{i} ; i=1, \ldots, n\right\}$ which on restriction are dual to $\Delta^{v} \otimes 1$. For $\omega \in \mathscr{I} \mathrm{nt}_{+}, P^{\omega}$ is $W$-invariant, and the multiplicity mult $t_{\omega}(\lambda)=\operatorname{mult}_{\omega}(w \lambda)$, $\forall w \in W, \forall \lambda \in P^{\omega}$. The root datum is "simply connected" if $\omega_{i} \in \mathfrak{h}_{\mathbf{Z}}^{*} \subseteq \mathfrak{h}^{*}, \forall i,[4,10]$.

1.3. Let the conjugate linear involution $\underline{\omega}_{0}$ on $\mathfrak{g}$ be given by $\underline{\omega}_{0}\left(e_{i}\right)=-f_{i}, \underline{\omega}_{0}\left(f_{i}\right)=$ $-e_{i}, i \in\{1, \ldots, n\}, \underline{\omega}_{0}(h)=-h, h \in \mathfrak{h}_{\mathbb{R}}:=\mathfrak{h}_{\mathbf{Z}} \otimes_{\mathbf{Z}} \mathbb{R}$. If $A$ is symmetrizable there is a hermitian form $(,)_{0}$ on $\mathfrak{g}$ by $(x, y)_{0}=-\left(x, \underline{\omega}_{0}(y)\right), x, y \in \mathfrak{g}$.

Define $V=\sum_{\omega \in f n t+n b^{*}}^{\oplus} V^{\omega}$, then for each $i \in\{1, \ldots, n\}$ the one parameter subgroups $U_{i}:=\left\{\exp c e_{i} ; c \in \mathbb{C}\right\}, \underline{\omega}_{0}\left(U_{i}\right)=\left\{\exp c f_{i} ; c \in \mathbb{C}\right\}$ generate a subgroup $G_{i} \leq \mathrm{GL}(V)$ isomorphic to $\operatorname{SL}(2, \mathbb{C})$. The algebraic torus $T:=\mathfrak{h}_{\mathbf{Z}} \otimes_{\mathbf{z}} \mathbb{C}^{*}$ has character group $\mathfrak{b}_{\mathbf{z}}^{*}$. With $\mathfrak{n}^{(i)}=$ $\sum_{a \in \Phi_{+}, b_{(z)>i} g_{\alpha}}^{\oplus} g$ let $U^{(i)}$ be the unipotent algebraic group with Lie algebra $n_{+} / n^{(i)}, i \in \mathbb{N}$. Let $U=\lim _{-} U^{(i)}$ the inverse limit, and $B=T U$ a semidirect product. Firally $G \leqq G L(V)$ is defined to be the group generated by $B$ and $G_{i}, i=1, \ldots, n$. The involution $\underline{\omega}_{0}$ lifts to $G$. There are monomorphisms $\phi_{i}: G_{i} \rightarrow G$ with $\phi_{i}\left\{\left(\begin{array}{ll}1 & c \\ 0 & 1\end{array}\right) ; c \in \mathbb{C}\right\}=U_{i}, i \in\{1, \ldots, n\}$, see [6].

Let $v_{\omega}$ be the highest weight vector of $V^{\omega}, \omega \in \mathscr{I} \mathrm{nt}+\cap \mathfrak{h}_{\mathbf{Z}}^{*}$. Now $B=\left\{g \in G ; g \sum_{\omega} \mathbb{C} v_{\omega}=\right.$ $\sum_{\omega} \mathbb{C} v_{\omega}$ (the Borel subgroup with Lie algebra $\mathfrak{b}$ ). We may regard the maximal torus 
$T=B \cap \underline{\omega}_{0}(B)$. Also let $N=N_{G}(T)$ the normalizer of $T$ in $G$. With $n_{i}:=\phi_{i}\left(\begin{array}{cc}0 & 1 \\ -1 & 0\end{array}\right)=$ $\left(\exp e_{i}\right)\left(\exp -f_{i}\right)\left(\exp e_{i}\right), i=1, \ldots, n$ and $N_{(1)}:=\left\langle n_{i} ; i=1, \ldots, n\right\rangle$, there is an exact sequence $1 \rightarrow T_{(2)} \rightarrow N_{(1)} \rightarrow W$, where $T_{(2)}:=\left\langle n_{i}^{2} ; i=1, \ldots, n\right\rangle, n_{i} \mapsto r_{i}$. Then $N=\left\langle T, N_{(1)}\right\rangle, T_{(2)}=N_{(1)} \cap$ $T=\left\{t \in G^{\prime} \cap T ; t^{2}=1\right\} \simeq \mathbb{Z}_{2}^{n}$, and $W \rightarrow N / T, r_{i} \mapsto n_{i} T$ is an isomorphism.

For any $\alpha \in \Phi_{+}^{\text {re }}$ let $i \in\{1, \ldots, n\}, w \in W$ be such that $w\left(\alpha_{i}\right)=\alpha$ and define root subgroup $U_{\alpha}=n U_{i} n^{-1}, n \in N, n T=w$. Each such $U_{\alpha}, \alpha \in \Phi_{+}^{\text {re }}$ is normalized by $T$ with $t u_{i}(c) t^{-1}=$ $u_{i}\left(\alpha_{i}(t) c\right), t \in T, c \in \mathbb{C}$ where $u_{i}(c):=\phi_{i}\left(\begin{array}{ll}1 & c \\ 0 & 1\end{array}\right)[5,9]$.

Let $U^{\mathrm{min}}=\left\langle U_{\alpha} ; \alpha \in \Phi_{+}^{\text {re }}\right\rangle$ and $B^{\mathrm{min}}=T U^{\mathrm{min}} \leqq B$. And $G^{\mathrm{min}}:=\left\langle T, G_{i} ; i=1, \ldots, n\right\rangle \leqq G$.

The group $G$ acts on $V^{\omega}$ by representation $R$, and also $G^{\min }$ acts on $g$ by the adjoint representation Ad. In fact if $(V, \phi)$ is a representation of $\mathfrak{g}$ such that the action of $\mathfrak{h}$ lifts to $T$ and $e_{\alpha}, f_{i}$ act locally finitely on $V, e_{\alpha} \in g_{\alpha} \hookrightarrow n_{+} / \mathfrak{n}^{(j)}, \mathrm{ht}(\alpha) \leqq j, \forall \alpha \in \Phi_{+}, \forall i, j$, then there is $(V, \mathrm{R})$ of $G$ satisfying (with exp: $\mathrm{g}_{f} \rightarrow G^{\mathrm{min}}$ the exponential mapping, having domain $\mathfrak{g}_{f}=\{y \in \mathfrak{g} ; y$ acts locally finitely on $\mathfrak{g}$ by ad $\left.\}\right), \mathbf{R}(\exp x)=\exp \phi(x), x \in \mathfrak{g}_{f}$. Thus $\phi=\mathrm{dR}$ the differential of $\mathrm{R}, \mathrm{ad}=\mathrm{d}(\mathrm{Ad})$. And $\mathrm{dR}(\operatorname{Ad}(g) x)=\mathrm{R}(g) \mathrm{dR}(x) \mathrm{R}(g)^{-1}, g \in G^{\min }, x \in g_{f}$.

We note that $R(n) V_{\lambda}=V_{w \lambda}$ and $\operatorname{Ad}(n) \mathfrak{g}_{\alpha}=\mathfrak{g}_{w(\alpha)}, \forall \lambda \in P^{\omega}, \forall \alpha \in \Phi$ where $n \in N, n T=w \in W$.

The group $G$ is said to have Lie algebra $g$ and is associated to the root datum $\left(\mathfrak{h}_{\mathbf{z}}, \Delta^{\vee}, \Delta\right)$.

The properties of a Tits system are satisfied. The group $G$ has $(B, N)$ pair with Coxeter group $W$. The Bruhat decomposition of $G$ into a disjoint union of double cosets of $B$ in $G$ is $G=\bigcup_{w \in W} B w B$; that is there is a bijection between the double cosets $B n B$ and $W$ under the natural epimorphism $N \rightarrow W$. Also to multiply double cosets

$$
\begin{aligned}
(B s B)(B w B) & =B s w B \text { if } l(s w)=l(w)+1 \\
& =B w B \cup B s w B \text { if } l(s w)=l(w)-1
\end{aligned}
$$

$w \in W, s=r_{i}, i \in\{1, \ldots, n\}$, where $l($.$) is the length function on W,[12]$.

1.4. Let $K=G^{\omega_{0}}$ the subgroup of fixed points of $\underline{\omega}_{0}$; this is called the "unitary form". The complex Iwasawa decomposition $G=K B$ holds [5]. Moreover $G^{\mathrm{min}}=K B^{\mathrm{min}}$.

From now on, unless stated otherwise, the superscript "min" will be omitted.

Proposition 1. Let $\alpha \in \Phi$ be such that the orbit $W . \alpha=\{\alpha\}$.

Then $\alpha \in \Phi^{\mathrm{im}}$ with $\alpha$ isotropic $((\alpha, \alpha)=0)$. And $\alpha$ as an element of the character group $\mathfrak{b}_{\mathbf{Z}}^{*}$ extends trivially to $\alpha \in \operatorname{Hom}\left(G, \mathbb{C}^{*}\right)$

Proof. Let $\alpha \in \Phi_{+}$with $w \alpha=\alpha, \forall w \in W$. As $w \alpha=\alpha_{i} \Rightarrow \alpha=\alpha_{i} \Rightarrow r_{i} \alpha=-\alpha_{i}=\alpha$, have $\alpha \in \Phi^{\mathrm{im}}$. Also $\alpha=\sum_{i \in \operatorname{supp} \alpha} c_{i} \alpha_{i} \Rightarrow(\alpha, \alpha)=\sum_{i \in \operatorname{supp} \alpha} c_{i}\left(\alpha, \alpha_{i}\right)=0$. The support of $\alpha, \operatorname{supp} \alpha$, is connected of affine type (see (2.1)).

In fact [4] conversely, $\alpha \in \mathbb{N} \Delta$, supp $\alpha$ connected and affine $\Rightarrow \alpha \in \Phi_{+}^{\text {im }}$ and $\alpha$ is isotropic with $w \alpha=\alpha, \forall w \in W$.

Let $G^{\prime}$ be the derived group of $G$. Decompose $T=T_{0} T_{1}$ with $T_{1} \cap G^{\prime}=\{1\}$ and $G=T_{1} G^{\prime}$ a semidirect product. Define for $w \in W, \Phi(w)=\left\{\alpha \in \Phi_{+} ; w^{-1} \alpha \in \Phi_{-}\right\}$and $U_{w}=$ $\prod_{\beta \in \Phi(w)} U_{\beta}$. There is a bijection $U_{w} \times B \rightarrow B n B=: C(w)$, where $n T=w$, by $(u, b) \mapsto u n b$.

As $\alpha$ is zero on $\Delta^{\vee}$ define $\alpha\left(G^{\prime}\right)=1$. We have $U<G^{\prime}, U \triangleleft B, G_{i} \leqq G^{\prime} \forall i, N_{(1)}<G^{\prime}$. Now 
$W$ acts on $T$ by $t \mapsto n t n^{-1}$. Therefore with $u n b=u n^{\prime} u_{1} t \in C(w), n^{\prime} \in N_{(1)}, u_{1} \in U, t \in T$ put $\alpha(u n b)=\alpha(t)$. To check that $\alpha$ is a homomorphism take $g_{1} \in C\left(w_{1}\right), g_{2} \in C\left(w_{2}\right)$; now $g_{1}=x_{1} t_{1}, g_{2}=x_{2} t_{2}, x_{j} \in B n_{j} B \cap G^{\prime}, j=1,2, t_{1}, t_{2} \in T_{1}$ gives $g_{1} g_{2}=x_{1}\left(t_{1} x_{2} t_{1}^{-1}\right) t_{1} t_{2}$.

Alternatively, after $T=T_{0} T_{1}$ one could observe that $\left(t_{1} g_{1}\right)\left(t_{2} g_{2}\right)=\left(t_{1} t_{2}\right)\left(t_{2}^{-1} g_{1} t_{2} g_{2}\right)$.

Proposition 2. Let $L_{1}=\left\langle T, U_{\alpha}, \underline{\omega}_{0}\left(U_{\alpha}\right) ; \alpha \in \Phi_{1+}\right\rangle$ be a Levi subgroup of $G$ of finite type $\Phi_{1+} \subseteq \Phi_{+}$.

Then $L_{1}=K_{1} T K_{1}$ where $K_{1}=L_{1}^{\prime} \cap B$.

Proof. It is clear from (1.3) that $L_{1}=K_{1} B_{1}, B_{1}=L_{1} \cap B$.

A real finite dimensional semisimple Lie algebra $g_{0}$ has Cartan subalgebras $\mathfrak{h}_{0}$, the set of which having finitely many conjugacy classes under the adjoint group Int $\mathrm{g}_{0}=\operatorname{Ad} G_{0}$, $\left(G_{0}\right.$ connected with Lie algebra $\left.g_{0}\right)$. If $g_{0}=\mathfrak{f}_{1} \oplus p$ is a Cartan decomposition with involution $\theta$, then under the action of the inner automorphisms Int $g_{0}$ we can assume that $\mathfrak{h}_{0}$ is $\theta$ stable. There are two extreme conjugacy classes; writing $\mathfrak{h}=\mathfrak{a}_{\mathfrak{l}_{1}} \oplus \mathfrak{a}_{\mathrm{p}}$ these are the fundamental class, when $a_{i_{1}}$ is maximal abelian in $\mathfrak{l}_{1}$, and the split class, when $a_{p}$ is maximal abelian in $\mathfrak{p}$. The pair $\left(\mathfrak{g}_{0}, \mathfrak{h}_{0}\right)$ gives root system $\Phi_{0}$, and with the split class $\left(g_{0}, a_{1}\right)$ the restricted root system $\Psi_{0}$. There is [13] the real Iwasawa decomposition

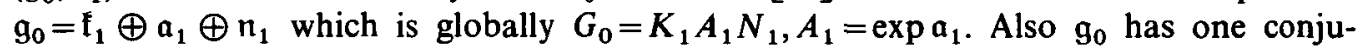
gacy class of Cartan subalgebras $\Leftrightarrow \mathfrak{a}_{\mathfrak{t}_{1}}$ is maximal abelian in $\mathfrak{f}_{1}$, (here $\mathfrak{h}_{0}=\mathfrak{a}_{\mathfrak{t}_{1}} \oplus \mathfrak{a}_{1}$ ). Since any two maximal abelian subalgebras in $\mathfrak{p}$ are conjugate under $K_{1}, \mathfrak{g}_{0}=$ $\mathfrak{f}_{1} \oplus \bigcup_{k \in K_{1}} \operatorname{Ad}(k) \mathfrak{a}_{1}$, and so $G_{0}=K_{1} A_{1} K_{1}$.

In our situation $K_{1}$ is maximal compact in $G_{0}:=K_{1 c} \leqq L_{1}$ and $\mathfrak{p}=\sqrt{-1} \mathfrak{f}_{1}$. Then (the centralizer of $a_{1}$ in $\left.\mathfrak{f}_{1}\right) m_{0}:=Z_{\mathfrak{t}_{1}}\left(a_{1}\right)=\sqrt{-1} a_{1}$ is a Cartan subalgebra of $\mathfrak{f}_{1}$. Thus $\Psi_{0}=\Phi_{0}$. And if $M_{0}:=Z_{K_{1}}\left(a_{1}\right)$, then $B_{0}=M_{0} A_{1}\left(\theta\left(N_{1}\right)\right)$ is a complex Lie subgroup of $G_{0}$, as $b_{0}=m_{0} \oplus a_{1} \oplus \theta \mathfrak{n}_{1}=m_{0 c} \oplus \sum_{\alpha \in \Phi_{0+}}^{\oplus} g_{-\alpha}$, and is closed.

The complex torus $T$ has Lie algebra $\mathfrak{h}$. And $T=T_{0} T_{1}$ with $T_{0} \leqq G_{0}$ having Lie algebra $\mathfrak{h}_{0}=\mathfrak{m}_{0 c}, \quad \mathfrak{h}=\mathfrak{h}_{0} \oplus \mathfrak{h}_{1}$. Then $I_{1}=\mathfrak{h}_{1} \oplus \mathfrak{g}_{0}$ with $\mathfrak{g}_{0}=\left[\mathfrak{l}_{1} \mathfrak{I}_{1}\right] \unlhd \mathfrak{I}_{1}$, and $G_{0}=$ $K_{1} T_{0} K_{1} \unlhd L_{1}, L_{1}=T_{1} G_{0}$ a semidirect product.

Let $T_{1 \mathbf{R}}$ be the 'real points' that is $\mathfrak{h}_{1 \mathbf{R}}=\left\{h \in \mathfrak{h}_{1} ; \alpha(h) \in \sqrt{-1} \mathbb{R} \forall \alpha \in \Phi_{1}\right\}$; here $T_{1}$ may not be central (see (3.1)). Now $\mathfrak{f}_{1}=\sqrt{-1} a_{1} \oplus \sum_{\alpha \in \Phi_{1}}^{\oplus} \mathbb{R} u_{\alpha}$ where $u_{\alpha}=\left(e_{\alpha}-e^{\alpha}\right)+$ $\sqrt{-1}\left(e_{\alpha}+e^{\alpha}\right)$ with $e_{\alpha} \in g_{\alpha},-e^{\alpha}:=\theta\left(e_{\alpha}\right) \in g_{-\alpha}, \alpha \in \Phi_{1+}$. We have $\left[h u_{\alpha}\right]=-\alpha(h) \sqrt{-1} u^{\alpha}$, $\forall h \in \mathfrak{h}_{1 R}$ and so, since $\operatorname{Ad}(\exp x)=\mathrm{e}^{\mathrm{ad} x}, \forall x \in \mathfrak{f}_{1}$ and each point of $K_{1}$ lies on a one parameter subgroup, then $\operatorname{Ad}(k) \mathfrak{h}_{1 \mathrm{R}} \subseteq \mathfrak{h}_{1 \mathrm{R}}+\mathfrak{f}_{1}, \forall k \in K_{1}$. Thus $\mathfrak{f}_{1}+\bigcup_{k \in K_{1}} \operatorname{Ad}(k)\left(\mathfrak{h}_{1 \mathrm{R}} \oplus\right.$ $\left.\mathfrak{h}_{0}\right)=\mathfrak{f}_{1} \oplus \mathfrak{h}_{1 \mathfrak{R}} \oplus \mathfrak{p} \leqq \mathfrak{I}_{1}$ over $\mathbb{R}$. Next as $T_{1 \mathbb{R}}$ is contained in the normalizer of $K_{1}$ in $L_{1}$ it follows that $K_{1} T_{0} T_{1 \mathrm{R}} K_{1} \leqq L_{1}$.

Hence over $\mathbb{C}, L_{1}=K_{1} T K_{1}$.

Note. For any subset $J \subseteq I=\{1, \ldots, n\}$ let $W_{J}=\left\langle r_{i} ; i \in J\right\rangle \leqq W$, and $N_{J}=\left\langle n_{i} ; i \in J\right\rangle \leqq N$. The conjugates in $G$ of $P_{J}=B N_{J} B$ are called the parabolic subgroups of $G$. Such a group $[1,11]$ has a Levi decomposition $P_{J}=L_{J} \ltimes U_{(J)}$ where $L_{J}$ is the Kac-Moody group associated to the root datum $\left(h_{z}, \Delta_{j}^{v}, \Delta_{J}\right)$ with $\Delta_{j}^{v}=$ $\left\{h_{i} ; i \in J\right\}, \Delta_{J}=\left\{\alpha_{i} ; i \in J\right\}$. The parabolic subgroup $P_{J}$ is said to be of finite type if $W_{J}$ is finite. 
The type of $G$ is defined according to the type of $A$ (with $A$ indecomposable, see (2.1)). We say (with $A$ possibly not symmetrizable) that $G$ is of type (3) if the orbits of $W$ acting on $\Phi_{+}^{\mathrm{im}}$ are not all singleton sets. The group $G$ is type (1) $\Leftrightarrow W$ is finite $\Leftrightarrow G$ is the homomorphic image of an almost simple, complex Lie group (with fundamental group $\left.\mathfrak{h}_{\mathbf{z}} / \mathbb{Z} \Delta^{\vee}\right)$.

Proposition 3. Let $G$ be of type (2) or (3). For each $\alpha \in \Phi^{\mathrm{re}}$ denote by $V_{m}, m \in \mathbb{N}$ the standard simple $G_{\alpha}=\phi_{\alpha}(\operatorname{SL}(2, \mathbb{C}))$ module; then $\left\{m \in \mathbb{N} ; V_{m} \leqq_{G \alpha} V^{\omega}\right\}$ is unbounded.

Proof. By $W$ conjugacy it suffices to prove this for a simple root $\alpha_{i}, i \in\{1, \ldots, n\}$. We have for type (1), (2), or (3) that $P^{\omega}=(\omega+\mathbb{Z} \Delta) \cap$ convex hull $(W . \omega)$, [4].

Type (2). The simple roots are (see (3.1)) labelled $\left\{\alpha_{0}, \alpha_{1}, \ldots, \alpha_{l}\right\}$. Let $\delta \in \mathfrak{h}^{*}$ be the positive imaginary root of least height. Then $\operatorname{supp}(\delta)=\{0,1, \ldots, l\}$ and $\Phi_{+}^{\mathrm{im}}=\{n \delta ; n \in \mathbb{N}\}$. Define maximal weights $\max (\omega)=\left\{\lambda \in P^{\omega} ; \lambda+\delta \in \mathfrak{h}^{*} \backslash P^{\omega}\right\}$. Then $P^{\omega}=\bigcup_{\lambda \in \max (\omega)}\{\lambda-$ $n \delta ; n \in \mathbb{N}\}$. The weight system lies in the paraboloid whose boundary intersects $P^{\omega}$ in the orbit $W . \omega$. Also $\max (\omega)$ consits of the highest weights of simple subquotients of $V^{\omega}$ under the action of Levi subgroups of $G$ of finite type.

Type (3). There exists a unique $\alpha \in \Phi_{+}^{\text {im }}$ of minimal height with $\operatorname{supp}(\alpha)=\{1, \ldots, n\}$ and $\alpha\left(h_{i}\right)<0, \forall i$. For $0 \neq v \in V_{\omega-\alpha}$ the mapping $n_{-} \rightarrow V^{\omega}, y \mapsto y . v$ is injective. As $\{j \alpha ; j \in \mathbb{N}\} \subseteq \Phi_{+}^{\mathrm{im}}$ we now have that $\{\omega-k \alpha ; k \in \mathbb{N}\} \subseteq P^{\omega}$, and $\forall i\left\{(\omega-k \alpha)\left(h_{i}\right) ; k \in \mathbb{N}\right\}$ is unbounded in $\mathbb{N}$.

Proposition 4. Let $L_{J}$ be a Levi subgroup of $G$ of finite type. Denote by $V_{J}^{\lambda}$ the simple $L_{J}$ module with highest weight $\lambda$. Then $\forall \omega \in \mathscr{I} \mathrm{nt}+\cap \mathfrak{h}_{\mathbf{Z}}^{*}$, the set $\left\{\lambda \in P^{\omega} ; V_{J}^{\lambda} \leqq_{L_{J}} V^{\omega}\right\}$ is infinite.

Proof. Any $\lambda \in P^{\omega}$ can be uniquely written $\lambda=\omega-\sum_{i \in I \backslash J} c_{i} \alpha_{i}-\sum_{i \in J} c_{i} \alpha_{i}$ where $c_{i} \in \mathbb{N}, \forall i$. Define $\operatorname{dep}_{J}(\lambda)=\sum_{i \in I \backslash J} c_{i}$. Then $V_{(m)}:=\sum_{\operatorname{dep}(\lambda) \leqq m}^{\oplus} V_{\lambda}, m \in \mathbb{N}$ is a finite dimensional $P_{J}$ submodule of $V^{\omega}$. And $V^{\omega}$ is completely reducible as an $L_{J}$-module. Thus $\left\{\operatorname{dep}_{J}(\lambda) ; \lambda \in P^{\omega}\right\}$ and $i \in J,\left\{m \in \mathbb{N} ; \exists \lambda \in P^{\omega}\right.$ with $\left.c_{i} \geqq m\right\}$ are unbounded.

The result is now a consequence of Proposition 3 .

If $i, j \in\{1, \ldots, n\}$ with $m=a_{i j} a_{j i} \geqq 2$ then label the $(i, j)$ edge in the Coxeter graph ${ }^{m}-$. It can be seen as in the examples affine $O 4-O \omega=\omega_{1}$, and hyperbolic $^{\alpha_{j}} \mathrm{O}-4-\mathrm{O}-\omega=\omega_{1}$ that in Proposition 4 the multiplicity $\operatorname{dim} \mathrm{Hom}_{L J}\left(V^{\infty}, V_{J}^{\lambda}\right)=0$ or $\infty$.

\section{Hilbert space structure and trace class operators}

2.1. Let $A$ be a symmetrizable Cartan matrix; so there is a positive rational matrix $D$ with $D^{-1} A$ symmetric. Then there are three types:

(1) $A$ has rank $n$ and $D^{-1} A$ has signature $n$

(2) $A$ has corank 1 and $D^{-1} A$ has signature $n-1$ 
(3) The signature of $D^{-1} A$ is less than the rank of $A$, of finite, affine and indefinite type respectively.

The simple quotient $V^{\omega}, \omega \in \mathscr{I}_{\mathrm{nt}}$ is, [6], a pre-Hilbert space via a contravariant, $K$-invariant, positive definite hermitian form $\langle$,$\rangle which is unique with norm \left\|v_{\omega}\right\|=1$. Order the weights $P^{\omega}$ by the depth, with $\omega$ minimal. Then $V_{\lambda} \perp V_{\mu}, \lambda \neq \mu, \lambda, \mu \in P^{\infty}$; and the completion also denoted by $V^{\omega}$ is separable. We fix an orthonormal basis $\left\{z_{i}\right\}_{i \in N}$ of $V^{\omega}$ where $z_{i}$ is of weight $\lambda_{i}, z_{0}=\omega$ and $\operatorname{dep}_{\omega}\left(\lambda_{i}\right) \geqq \operatorname{dep}_{\omega}\left(\lambda_{j}\right), i \geqq j$.

In the representation $\left(V^{\omega}, \mathrm{R}\right)$ of $G=G(A)$ we will say that an operator $\mathrm{R}(g), g \in G$ is traceable if the complex series $\sum_{i=0}^{\infty}\left\langle\mathrm{R}(g) z_{i}, z_{i}\right\rangle$ is convergent; then this value is written $\operatorname{trace}_{\omega} R(g)$.

2.2. As in a general separable Hilbert space, let $\mathbb{B d}(V), \operatorname{Fr}(V), \mathbb{K p}(V), \operatorname{St}(V)$ and $\operatorname{Tr}(V)$ be the set of bounded linear, finite rank, compact, Hilbert-Schmidt and traceable (with absolute convergence) operators on $V$. That is $\operatorname{St}(V)=\left\{T \in \operatorname{End}(V) ;\|T\|_{2}<\infty\right\}$ where $\|T\|_{2}=\sum_{i}\left\|T z_{i}\right\|^{2}$ (the $T \in \operatorname{St}(V)$ are $l^{2}$ ). And $\operatorname{Tr}(V)=\left\{T \in \operatorname{End}(V) ; \sum_{i}\left|\left\langle T z_{i}, z_{i}\right\rangle\right|<\right.$ $\infty\}$. In fact $\mathbb{S t}(V) \subseteq \mathbb{B d}(V)$ and $\left(\mathcal{S t}(V),\|\cdot\|_{2}\right)$ is a Banach * algebra. A $T \in \operatorname{Tr}(V)$ may not be bounded. For $T \in S t(V)$, the Hilbert-Schmidt norm is independent of the complete orthonormal basis. Then $\mathbb{K} \mathrm{p}(V)$ is the unique maximal ideal in $\mathbb{B d}(V)$ which is closed in the operator norm; and $\operatorname{Fr}(V)$ is the unique minimal ideal in $\operatorname{Bd}(V)$. The ideal $\operatorname{St}(V)$ is not closed. In fact $\mathbb{K} \mathrm{p}(V)=\overline{\mathbb{F r}(V)}$.

One says that $T \in \mathbb{B d}(V)$ is $l^{1}$ if $\sum_{i}\left\|T z_{i}\right\|<\infty$; in fact $T$ is $l^{1} \Leftrightarrow T \in \operatorname{St}(V)^{2}$. Then $\operatorname{St}(V)^{2} \subseteq \mathbb{B d}(V) \cap \operatorname{Tr}(V)$ and $\operatorname{trace}(T), T \in \mathbb{S t}(V)^{2}$ is independent of the orthonormal basis. Also trace $(S T)=\operatorname{trace}(T S)$ for $S \in \mathbb{B d}(V), T \in S \mathrm{t}(V)^{2}$. These give a chain of (two sided) ideals

$$
\{0\} \subseteq \mathbb{F} \mathrm{r}(V) \subseteq \mathrm{St}(V)^{2} \subseteq \mathrm{St}(V) \subseteq \mathbb{K} \mathrm{p}(V) \subseteq \mathbb{B d}(V)
$$

A $T \in \operatorname{End}(V)$ is said to be closed if its graph is closed in $V \times V$; and closeable if $\overline{\operatorname{graph}(T)}$ is a graph. If $T$ is closeable then there is a unique $\bar{T} \in \operatorname{End}(V)$ with $\operatorname{graph}(\bar{T})=\operatorname{graph}(T)$; the domain being $\operatorname{dom}(\bar{T})=\left\{x \in V ; \exists\right.$ sequence $\left(x_{n}\right)$ in $\operatorname{dom}(T)$ with $x_{n} \rightarrow x$ and $\left(T x_{n}\right)$ convergent $\}$, and $T x=\lim T x_{n}$. A $T \in \operatorname{End}(V)$ is said to be hermitian if it is a formal adjoint of itself, and symmetric if it is hermitian and densely defined.

2.3. Subsets of $G$ are defined

$$
\begin{gathered}
G^{\mathbf{b}}=\left\{g \in G ; \mathrm{R}(g) \in \mathbb{B d}\left(V^{\omega}\right), \forall \omega \in \mathscr{I} \mathrm{nt}_{+} \cap \mathfrak{h}_{\mathbf{z}}^{*}\right\} \\
G^{\mathrm{tr}}=\left\{g \in G ; \mathrm{R}(g) \text { is traceable on } V^{\omega}, \forall \omega \in \mathscr{I} \mathrm{nt}_{+} \cap \mathfrak{h}_{\mathbf{z}}^{*}\right\}
\end{gathered}
$$

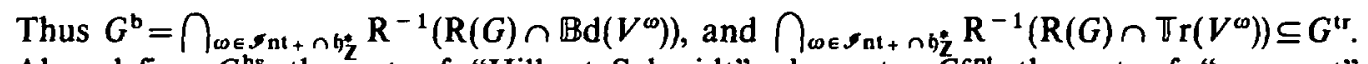
Also define $G^{\text {hs }}$ the set of "Hilbert-Schmidt" elements, $G^{\text {cpt }}$ the set of "compact" elements, $G^{\text {fr }}$ the set of "finite rank" elements, giving $G^{\mathrm{fr}} \subseteq\left(G^{\mathrm{hs}}\right)^{2} \subseteq G^{\text {hs }} \subseteq G^{\mathrm{cpt}} \subseteq G^{\mathrm{b}}$. And $G^{\text {sym }}$ the set "symmetric" elements, $G^{\text {cl }}$ the set of "closeable" elements. 
Lemma 1. (i) $K G^{\mathrm{s}} K=G^{\mathrm{s}}$ where $G^{\mathrm{s}}$ is the semigroup $G^{\mathrm{b}}, G^{\mathrm{cpt}}, G^{\mathrm{hs}}$ or $\left(G^{\mathrm{hs}}\right)^{2}$.

(ii) $G^{\mathrm{fr}}=\emptyset$ if $A$ is not of type (i).

Proof. (i) This follows from $\mathrm{R}(K) \subseteq U\left(V^{\omega}\right), \forall \omega$ (the unitary group).

(ii) The Iwasawa decomposition $G=K B$ gives $G^{\mathrm{fr}}=K B^{\mathrm{fr}}$. Further $\mathrm{R}(b) V_{\lambda} \subseteq$ $\sum_{\mu \in P^{\omega}, \mu \leqq \lambda}^{\oplus} V_{\mu}$ with $\left\langle R(b) V_{\lambda}, V_{\lambda}\right\rangle \neq 0, \forall b \in B, \forall \lambda \in P^{\omega}$. Hence $B^{\mathrm{fr}}=\emptyset$.

Proposition 5. Let $A$ be of type (2) or (3). Then

$$
U \cap G^{\mathrm{b}}=\{1\}
$$

Proof. For each $\alpha_{i} \in \Delta$ there is the Levi subgroup $L_{\{i\}}$ of the parabolic subgroup $P_{\{i\}}$ of $G$ (see (1.4)) $i \in\{1, \ldots, n\}$. And if $\alpha \in \Phi^{\text {re }}$ with $w \in W, w\left(\alpha_{i}\right)=\alpha$ and $n \in N, n \mapsto w \in N / T$, we have $L_{\alpha}=n L_{\{i\}} n^{-1}=\left\langle T, U_{\alpha}, \underline{\omega}_{0}\left(U_{\alpha}\right)\right\rangle$. The derived group $L_{\alpha}^{\prime} \simeq \operatorname{SL}(2, \mathbb{C})$. The simple $G$-module $V^{\omega}, \omega \in \mathscr{I}_{\mathrm{nt}} \cap \cap \mathfrak{h}_{2}^{*}$ is semisimple under $L_{\alpha}$, which is such that this decomposition under $\phi_{\alpha}(S U(2))$ is a complete orthogonal direct sum.

Also recall that $\operatorname{GL}(2, \mathbb{C})$ acts on $\bigvee^{m}\left(\mathbb{C}^{2}\right)$, the symmetric polynomials of degree $m$ in $X, Y$, by $(g \cdot p)\left(\begin{array}{l}X \\ Y\end{array}\right)=p\left(g^{\mathrm{t}}\left(\begin{array}{l}X \\ Y\end{array}\right)\right)$. The standard basis vectors are

$$
Z=\left(\frac{1}{a ! b !}\right)^{\frac{1}{2}} X^{\mathrm{a}} Y^{\mathrm{b}}, a+b=m
$$

and the unipotent element $u=u(c)=\left(\begin{array}{ll}1 & c \\ 0 & 1\end{array}\right), c \in \mathbb{C}$, acts as

$$
\text { u. } Z=\sum_{r=0}^{b} c^{r}\left(\begin{array}{l}
b \\
r
\end{array}\right)\left(\frac{(a+r) !(b-r) !}{a ! b !}\right)^{\frac{1}{2}} \frac{X^{a+r} Y^{b-r}}{((a+r) !(b-r) !)^{\frac{1}{2}}}
$$

The superdiagonal entries are with $r=1, c(a+1)^{\frac{1}{2}} b^{\frac{1}{2}}$ which with $a=0, b=m$ is $\mathrm{cm}^{\frac{1}{2}}$. Label the weight vectors

$$
z_{0}=Y^{m}, z_{1}=\left(\frac{1}{(m-1) !}\right)^{\frac{1}{2}} X Y^{m-1}, \ldots, z_{m}=X^{m}
$$

with weights $-m, 2-m, \ldots, m$ under $h_{i}$. This $\left\{\bigvee^{m}\left(\mathbb{C}^{2}\right) ; m \in \mathbb{N}\right\}$ is a complete set of simple finite dimensional $\operatorname{SL}(2, \mathbb{C})$-modules.

Let $u=u_{1} \cdots u_{k} \in U, u_{j}=u_{j}\left(c_{j}\right)$ with $c_{j} \neq 0$ some $j$ and for each $j \in\{1, \ldots, k\}$ we have $u_{j} \in U_{\beta_{j}}, \beta_{j} \in \Phi_{+}^{\text {re }}$. There is $w_{1} \in W$ with $w_{1}\left(\beta_{1}\right)=\alpha_{i}$ for some $i \in\{1, \ldots, n\}$. Let $K_{i}=$ $\phi_{i}(\operatorname{SU}(2)), i \in\{1, \ldots, n\}$. As a product of fundamental reflections $w_{1}=r_{i_{1}} \cdots r_{i_{t}}$ say, so taking conjugates $u, \quad n_{i_{t}} u n_{i_{t}}^{-1}, \quad n_{i_{t-1}} n_{i_{t}} u n_{i_{t}}^{-1} n_{i_{t-1}}^{-1}, \ldots$, where $n_{i_{j}} \mapsto r_{i_{j}} \in N / T, \quad n_{i_{j}}=$ $\phi_{i_{j}}\left(\begin{array}{cc}0 & 1 \\ -1 & 0\end{array}\right) \in K_{i j}$, and using the fact that $\forall i^{\prime},\left(r_{i^{\prime}}(\alpha) \in \Phi_{+}^{\text {re }}, \forall \alpha \in \Phi_{+}^{\text {re }}, \alpha \neq \alpha_{i}\right)$, we stop this sequence when a conjugate of $u$ contains a term in the product belonging to a simple 
root subgroup. Therefore we may as well start with $u=u_{1} \cdots u_{k}$ such that $\beta_{j}=\alpha_{i}$ some $i, j$.

Let such $\left\{z_{0}, \ldots, z_{m}\right\}$ with weights $\left\{\lambda_{0}, \lambda_{1}, \ldots, \lambda_{m}\right\}$ refer to a simple module in the $L_{\alpha_{i}}$ decomposition of $V^{\omega}$. We have

$$
\begin{aligned}
\mathbf{R}(u) z_{0} & =\mathbf{R}\left(u_{(2)}\right)\left(z_{0}+z\right) \text { if } \beta_{k} \neq \alpha_{i} \\
& =\mathbf{R}\left(u_{(2)}\right)\left(z_{0}+c_{k} m^{\frac{1}{2}} z_{1}+z\right) \text { if } \beta_{k}=\alpha_{i}
\end{aligned}
$$

where $u_{\left(k^{\prime}\right)}=u_{1} \cdots u_{k-\left(k^{\prime}-1\right)}$ and $z$ is a sum of weight vectors (or zero) with weights $\lambda_{0}+r \beta_{k}$, and $r \geqq 1$ if $\beta_{k} \neq \alpha_{i}$ or $r \geqq 2, \lambda_{1}=\lambda_{0}+\alpha_{i}$ if $\beta_{k}=\alpha_{i}$. Next $R(u) z_{0}=$ $R\left(u_{(3)}\right) R\left(u_{k-1}\right) R\left(u_{k}\right) z_{0}$ etc. to obtain finally

$$
\mathrm{R}(u) z_{0}=z_{0}+\sum_{j, \beta_{j}=z_{i}} c_{j} m^{\frac{1}{2}} z_{1}+z^{\prime} \text { and }\left\langle R(u) z_{0}, z_{1}\right\rangle=\sum_{\beta_{j}=\alpha_{i}} c_{j} m^{\frac{1}{2}}
$$

The result now follows immediately from Proposition 3 if $\sum_{\beta_{j}=\alpha_{i}} c_{j} \neq 0$.

Otherwise proceed as follows. The $L_{\beta_{j}}$ decomposition of $V^{\omega}$ is such that

$$
\mathrm{R}\left(u_{j}\left(c_{j}\right)\right) z_{\mathrm{a}}=\sum_{r=0}^{\mathrm{b}} c_{j}^{\mathrm{r}}\left(\begin{array}{c}
a+r \\
r
\end{array}\right)^{\frac{1}{2}}\left(\begin{array}{l}
b \\
r
\end{array}\right)^{\frac{1}{2}} z_{a+r}, a+b=m .
$$

Again under $L_{\alpha_{i}}$, the matrix elements $m_{l^{\prime} l^{\prime \prime}}(u)$ of $\mathrm{R}(u)$ are polynomials in the $c_{j}, j \in\{$ $1, \ldots, k\}$ with positive integer coefficients. Then, and using convex properties of $P^{\omega}$ described in (1.4), one sees that $u \in G^{\mathbf{b}} \Rightarrow \forall l^{\prime}, l^{\prime \prime}$ each polynomial in $m_{l^{\prime} l^{\prime \prime}}(u)$ which involves and is homogeneous in the $c_{j}, \beta_{j}=\alpha_{i}$ must be zero. Thus $u \in G^{\mathrm{b}} \Rightarrow u=u^{(i)}$ (obtained from $u$ be deleting the $u_{j} \in U_{\alpha_{i}}$ ). Continuing, up to conjugation by $N \cap K$ the element $u^{(i)}$ has $u_{j} \in U_{a_{i}}$, for some $i^{\prime}, j$. Finally, $u \in G^{b} \Rightarrow u=1$.

To make the previous section more precise we include the following auxiliary results:

Let [ ] denote the group commutator, that is $[x, y]=x^{-1} y^{-1} x y, x, y \in G$. Define inductively $\left[x_{1}, \ldots, x_{m}\right]:=\left[\left[x_{1}, \ldots, x_{m-1}\right], x_{m}\right], m>2, x_{i} \in G$. Sometimes we denote $x^{y}:=y^{-1} x y$, therefore $x^{y}=x[x, y], x, y \in G$.

Here $A$ need not be symmetrizable. Recall that $U=U^{\text {min }}$.

Lemma 5a. If $W \ni w=r_{i_{1}} \cdots r_{i_{3}}$ is a reduced expression (where $r_{i_{j}}=r_{a_{i j}}$ ) then

$$
\Phi(w)=\left\{\alpha_{i_{1}}, r_{i_{1}}\left(\alpha_{i_{2}}\right), \ldots, r_{i_{1}} \cdots r_{i_{s-1}}\left(\alpha_{i_{s}}\right)\right\}
$$

In particular $l(w)=|\Phi(w)|$.

For $w \in W$, let $U_{w}=\prod_{\beta \in \Phi(w)} U_{\beta}$; this expresses an element uniquely as a product.

Proof. See for example [1].

Lemma 5b. Let $w_{1}, w_{2} \in W, \Phi\left(w_{1}\right) \cap \Phi\left(w_{2}\right)=\emptyset$. Then 
$w \in W, \Psi \subseteq \Phi_{+}, w \Psi \subseteq \Phi_{+} \Rightarrow\left(w\left(\Psi \cap \Phi\left(w_{1}\right)\right) \subseteq \Phi\left(w w_{1}\right)\right.$ and $\left.w \Psi \cap \Phi\left(w w_{1}\right) \cap \Phi\left(w w_{2}\right)=\emptyset\right) . \nabla$

Let $W_{(0)}=\{w \in W ; \Phi(w) \cup-\Phi(w)$ is a closed subsystem of roots in $\Phi\}$ and $N_{(0)}=$ $\left\{n \in N ; n \mapsto w \in W_{(0)}\right\}$.

Lemma 5c. Let $w \in W_{(0)}, \beta, \beta^{\prime} \in \Phi(w), \gamma \in \Phi_{+}^{\text {re }} \backslash \Phi(w)$. Then

$$
\begin{gathered}
{\left[U_{\beta}, U_{\gamma}\right] \leqq U \cap n U n^{-1}, N \ni n \mapsto w} \\
{\left[\left[U_{\beta}, U_{\gamma}\right], U_{\beta^{\prime}}\right] \leqq U \cap n U n^{-1} .}
\end{gathered}
$$

Proof. Take $w=r_{i_{1}} \cdots r_{i_{m}}$ a reduced expression. First consider $\beta=\alpha_{i_{1}}$. The $+\alpha_{i_{1}}$ chain of roots through $\gamma$ is $C_{i_{1}, \gamma}=\Phi \cap\left\{\gamma+s \alpha_{i_{1}} ; s \in \mathbb{N}\right\}$. Using the $L_{\alpha_{i_{1}}}$ decomposition of $\mathfrak{g}$ we see that $C_{i_{1}, \gamma}$ is finite and "unbroken". Let $x_{i_{1}}(c)=\exp c e_{i_{1}}, x_{\gamma}(c)=\exp c e_{\gamma}$ (where $e_{\gamma}=\operatorname{Ad}\left(n^{\prime}\right) e_{\alpha_{i^{\prime}}}, n^{\prime} \mapsto w^{\prime}, w^{\prime-1}(\gamma)=\alpha_{i^{\prime}}$, for chosen $\left.w^{\prime}, \alpha_{i}\right)$. We have

$$
\begin{aligned}
{\left[x_{\gamma}(c), x_{i_{1}}\left(c_{1}\right)\right]=} & x_{\gamma}(-c) x_{i_{1}}\left(-c_{1}\right) x_{\gamma}(c) x_{i_{1}}\left(c_{1}\right) \\
= & x_{\gamma}(-c) \exp \operatorname{Ad}\left(x_{i_{1}}\left(-c_{1}\right)\right)\left(c e_{\gamma}\right) \\
= & x_{\gamma}(-c) \exp \left(e^{-c_{1} \text { ad } e_{i_{1}}}\left(c e_{\gamma}\right)\right) \\
= & x_{\gamma}(-c) \exp \left(c \left(e_{\gamma}-c_{1}\left[e_{i_{1}} e_{\gamma}\right]+\frac{c_{1}^{2}}{2}\left[e_{i_{1}}\left[e_{i_{1}} e_{\gamma}\right]\right]\right.\right. \\
& \left.-\frac{c_{1}^{3}}{3 !}\left[e_{i_{1}}\left[e_{i_{1}}\left[e_{i_{1}} e_{\gamma}\right]\right]\right]+\ldots\right)
\end{aligned}
$$

a finite series

$$
\left(=1 \text { if } \gamma+\alpha_{i_{1}} \in \mathscr{I} \mathrm{nt} \backslash \Phi\right) .
$$

Next $\Phi(w)$ is a system of positive roots for a semisimple Lie subalgebra of $\mathrm{g}$, with Cartan subalgebra contained in $\mathfrak{h}$. Also mult $\alpha=1, \forall \alpha \in \Phi^{\text {re }}$. It follows that

$$
\gamma+s \alpha_{i_{1}} \in \Phi(w) \Rightarrow 0 \neq\left[f_{i_{1}} \ldots\left[\begin{array}{c}
{\left[f_{i_{1}}\left[f_{i_{1}} e_{\gamma+s a_{1}}\right]\right.} \\
s \text { times }
\end{array}\right] \ldots\right] \in \Phi(w) \Rightarrow \gamma \in \Phi(w)
$$

We conclude that $C_{i_{1}, \gamma} \cap \Phi_{+} \subseteq \Phi_{+} \backslash \Phi(w)$. Hence $n^{-1}\left[x_{\gamma}(c), x_{i_{1}}\left(c_{1}\right)\right] n \in U$.

Secondly, with any $\beta \in \Phi(w)$, use induction on $l(w)$. Suppose $l(w)=1, w=r_{i_{1}}$. Therefore $\beta=\alpha_{i_{1}}$. We want to show $\left[U \alpha_{i_{1}}, U_{\gamma}\right] \leqq U \cap n_{i_{1}} U n_{i_{1}}^{-1}$, which follows from (†). Suppose $l(w)=m>1$. Again by the first part we need only consider $\beta \neq \alpha_{i_{1}}$. Therefore 
$r_{i_{1}}(\beta) \in \Phi\left(r_{i_{1}} w\right), r_{i_{1}}(\gamma) \in \Phi_{+}^{\text {re }} \backslash \Phi\left(r_{i_{1}} w\right)$. Thus if we have the assertion for length $=m-1$, it follows that

$$
n_{i}\left[U_{\beta} U_{\gamma}\right] n_{i}^{-1}=\left[U_{r_{i},(\beta)} U_{r_{i 1}(\gamma)}\right] \leqq U \cap n_{i_{1}} n U n^{-1} n_{i_{1}}^{-1}
$$

which on conjugation by $n_{i_{1}}$ gives the result.

From $(\dagger)$ with $\alpha_{i_{1}}$ replaced by $\beta$ and using the commutator formula $[x y, z]=$ $[x z]^{y}[y z], x, y, z \in G$, we see by a similar argument that

$$
\left\{(\gamma+s \beta)+s^{\prime} \beta^{\prime} ; s, s^{\prime} \in \mathbb{N}\right\} \cap \Phi_{+} \subseteq \Phi_{+} \backslash \Phi(w)
$$

and

$$
\left[\left[x_{\gamma}(c), x_{\beta}\left(c_{1}\right)\right], x_{\beta^{\prime}}\left(c^{\prime}\right)\right] \in U \cap n U n^{-1}, \forall c, c_{1}, c^{\prime} \in \mathbb{C}
$$

as required.

Proposition 5d. $U=U_{w} \ltimes\left(U \cap n U n^{-1}\right), N \ni n \mapsto w \in W_{(0)}, \forall w \in W_{(0)}$.

Proof. A $u \in U$ can be expressed $u=u_{0} u_{1} \cdots u_{k}$ where $u_{0} \in U_{w}$ and $\forall j\left(u_{j} \in U_{w_{j}}\right.$, $\Phi(w) \cap \Phi\left(w_{j}\right)=\emptyset$ or $\exists \beta_{j} \in \Phi(w), \gamma_{j} \in \Phi_{+}^{\text {re }} \backslash \Phi(w)$ with $\left.u_{j} \in\left[U_{\beta_{j}} U_{\gamma_{j}}\right]^{U_{w}}\right)$.

Let $j \in\{1, \ldots, k\}$ with $u_{j} \in U_{w_{j}}$ and $\Phi(w) \cap \Phi\left(w_{j}\right)=\emptyset$. Then $U_{\beta} \leqq W_{w_{j}} \Rightarrow w^{-1} \beta=\alpha \in \Phi_{+}^{\text {re }} \Rightarrow$ $\beta=w \alpha \Rightarrow U_{\beta}=n U_{\alpha} n^{-1}$. Thus also using Lemmas 5a, 5c, we have $U=U_{w}\left(U \cap n U n^{-1}\right)$. Also $U_{w} \cap n U n^{-1}=\{1\}$, and with $u \in U \cap n U n^{-1}, v \in U_{\alpha}, \alpha \in \Phi(w)$ it follows that $u^{v}=$ $u_{1}^{v} \cdots u_{k}^{v}=u_{1}\left[u_{1} v\right] \cdots u_{k}\left[u_{k} v\right] \in U \cap n U n^{-1}$. Hence $U \cap m U m^{-1} \triangleleft U, \forall m \in N_{(0)}$.

Lemma 5e. Let $U_{(0)}=\bigcap_{n \in N_{(0)}} U \cap n U n^{-1}$ and $U_{(00)}=\bigcap_{n \in N} U \cap n U n^{-1}$. Then $U_{(0)} \checkmark$ $U, U_{(00)} \leqq U^{\prime}$ and any $u \in U_{(00)}$ can be expressed $u=u_{1} \cdots u_{k}$ with each $u_{j}$ of the form $x=\left[x_{1}, \ldots, x_{m}\right], x_{j^{\prime}} \in U_{\beta_{j^{\prime}}}, \beta_{j^{\prime}} \in \Phi_{+}^{\text {re }}$ or $x^{-1}$ and $\forall j, u_{j} \in U_{(0)}$.

Proof. Let $u \in U_{(00)}$. First write $u=u^{\prime} u^{\prime \prime}$ with $u^{\prime \prime} \in U^{\prime}$. Now $u^{\prime}=v_{1} \cdots v_{k^{\prime}}$ a product of elements of $U$ each lying in real root subgroups. Similarly $u^{\prime \prime}$ can be so expressed. If there are $i, j$ with $v_{j} \in U_{\alpha_{i}}$, then using Proposition 5d, we can reexpress $u=v^{\prime} v^{\prime \prime}$ where $v^{\prime \prime} \in U^{\prime}$ and $v^{\prime}$ is the product of $\leqq k^{\prime}-1$ elements of $U$ lying in real root subgroups. Otherwise, there is a sequence $\left(i_{1}, i_{2}, \ldots, i_{m^{\prime}}\right)$ and an $i$ such that $\left\{u^{\prime n}, u^{\prime \prime \prime}\right\} \subseteq U$ and the $\alpha_{i}$ root subgroup contains an element occurring in $u^{\prime n}$ (see the first part of the proof), where $N \cap K \ni n \mapsto w=r_{i_{1}} r_{i_{2}} \cdots r_{i_{m^{\prime}}}$. Now $u^{n} \in U_{(00)}$, also if $k^{\prime}=1$ we must have $v_{1}=1$. Hence by induction on $k^{\prime}, u \in U^{\prime}$. And $U_{(0)}$ the intersection of normal subgroups, is therefore normal in $U$.

Although $U$ is not locally nilpotent in type (2) or (3), the lower central series gives that $u=v_{1} \cdots v_{k^{\prime}}$ with each $v_{j}$, of the form $x=\left[x_{1}, \ldots, x_{m}\right]$ or $x^{-1}$ as in the statement of the result. Next

$$
\exists n \in N, n^{-1} x n \in G \backslash U \Rightarrow \exists j, n^{-1} x_{j} n \in G \backslash U \Rightarrow \beta_{j} \in \Phi(w), n \mapsto w \in W .
$$


If $\exists j^{\prime}, j^{\prime \prime} \in\{1, \ldots, m\}, m \geqq 2$ with $\beta_{j^{\prime}} \in \Phi(w), \beta_{j^{\prime \prime}} \in \Phi_{+}^{\text {re }} \backslash \Phi(w)$ for $w \in W_{(0)}$, then Lemma 5 , Proposition 5d and induction on $m$ give $n^{-1} x n \in U$. Thus for $n \in N_{(0)}, n^{-1} x n \in G \backslash U \Leftrightarrow \forall j$, $\beta_{j} \in \Phi(w)$. Set $I=\left\{1, \ldots, k^{\prime}\right\}, I_{1}=\left\{j \in I ; \exists n \in N_{(0)}, n^{-1} v_{j} n \in G \backslash U\right\}$. Then $j \in I_{1}, j^{\prime} \in I \backslash I_{1} \Rightarrow$ $\left[v_{j} v_{j^{\prime}}\right] \in U_{(0)}$ and can be written in the required form. Finally using $U \cap \underline{\omega}_{0}(U)=\{1\}$ we see that the result follows.

Note that $x \in U_{(00)} \Rightarrow \sum_{j=1}^{m} \mathbb{Z} \beta_{j} \cap \Phi^{\mathrm{im}} \neq \emptyset$.

If $w=w_{1} w_{2} \in W$ where $l(w)=l\left(w_{1}\right)+l\left(w_{2}\right)$, then $\Phi\left(w_{1}\right) \subseteq \Phi(w)$ and $(\dagger)$ (with $\alpha_{i_{1}}$ replaced by $\left.\beta \in \Phi(w), \gamma \in \Phi_{+}^{\text {re }} \backslash \Phi(w)\right)$ give that $w \in W_{(0)}$ implies $U \cap n U n^{-1} \leqq U \cap n^{\prime} U n^{\prime-1}, N \ni n \mapsto$ $w, N \ni n^{\prime} \mapsto w_{1}$.

Let $w \in W$, and $u=u_{0} u_{1} \cdots u_{k}$ with $u_{0} \in U_{w}$, and $u_{j}, j \neq 0$ as in the proof of Proposition $5 \mathrm{~d}$. From Lemma $5 \mathrm{a}$ we can further write uniquely $u_{0}=u_{01} \cdots u_{0 m}$ with $u_{0 s} \in U r_{i_{1}} \ldots r_{i_{s-1}}\left(\alpha_{i_{s}}\right), m=l(w)$. Suppose that $u \in G^{b}$. Then as in the first part of the proof we see that $u_{01}=1$. Next let $u_{02}=\cdots=u_{0, s-1}=1$ and put $w_{1}=r_{i_{1}} \cdots r_{i_{s-1}}, s \leqq m$. Now $w_{1} \Phi\left(w_{1}^{-1} w\right) \subseteq \Phi(w)$, Lemma $5 \mathrm{~b}$ and (†) give that $w \in W_{(0)}, N \cap K \ni n^{\prime} \mapsto w_{1}, u^{n^{\prime}} \in G^{\mathrm{b}} \Rightarrow$ $u_{0 s}=1$. Thus $u_{0}=1$. And as this holds $\forall w \in W_{(0)}$, we have shown $U \cap G^{\mathrm{b}} \subseteq U_{(0)}$. Note that in general one has $U=U_{w}\left(U \cap n U n^{-1}\right)$ for any $w \in W$. In fact for $w \in W$, use induction on $l(w)$. Suppose $u^{n^{\prime}} \in U$. Then as $u_{0}^{n^{\prime}} \in U$ we have $\left(u_{1} \ldots u_{k}\right)^{n^{\prime}} \in U$ giving $u_{0 s}=1$.

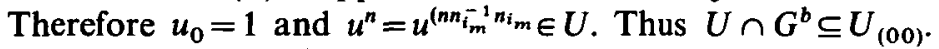

Let $u \in U_{(00)} \cap G^{\mathrm{b}}$. From Lemma 5e we write $u=u_{1} u^{\prime}, u_{1}=x=\left[x_{1}, \ldots, x_{m}\right]$ or $u_{1}=x^{-1}$. And show $u_{1}=1$. This is by induction on $m$.

If $m=2, x=\left[x_{\beta_{1}}\left(c_{1}\right), x_{\beta_{2}}\left(c_{2}\right)\right]$ and refer to $(\dagger)$. We can assume $\beta_{1}+\beta_{2} \in \Phi_{+}$. Note as before that $\lambda, \mu \in P^{\omega},\left\langle\mathrm{R}(x) V_{\lambda}, V_{\mu}\right\rangle \neq\{0\} \Rightarrow \mu=\lambda+s_{1} \beta_{1}+s_{2} \beta_{2}, s_{1}, s_{2} \in \mathbb{N} \backslash\{0\}$. Consider $C_{\beta_{2}, \beta_{1}} \cap \Phi_{+}^{\text {re }}$ and recall $W \Phi_{+}^{\text {im }}=\Phi_{+}^{\text {im }}$. If the $+\beta_{2}$ chain of roots through $\beta_{1}$ contains at least two real roots then $\exists w \in W, \exists s \in \mathbb{N} \backslash\{0\}, w^{-1}\left(C_{\beta_{2}, \beta_{1}} \backslash\left\{\beta_{1}\right\}\right) \subseteq \Phi_{+}$and $w^{-1}\left(\beta_{1}+s \beta_{2}\right)=$ $\alpha_{i} \in \Delta$. (Also $w^{-1} \beta_{1} \in \Phi_{+}$if $\Phi\left(r w_{i}\right)$ is a system of positive roots). Otherwise $C_{\beta_{2}, \beta_{1}} \cap \Phi_{+}^{\text {re }}=$ $\left\{\beta_{1}\right\}$ which is false. Thus also using $u^{\prime} \in U^{\prime}$, we have $u_{1}=1$.

For the induction step, $x=\left[\left[x_{1}, \ldots, x_{m-1}\right] x_{m}\right]=\left[x_{1}, \ldots, x_{m-1}\right]^{-1}\left[x_{1}, \ldots, x_{m-1}\right]^{x_{m}}$. Firstly, suppose $\exists w \in W_{(0)}$ with $u_{2}:=\left[x_{1}, \ldots, x_{m-1}\right] \in U_{w}$. Now (see Lemma 5a)

$$
u_{2}=y z, y, z \in U_{w},\left[y z, x_{m}\right]=\left[y x_{m}\right]^{z}\left[z x_{m}\right]=\left[y x_{m}\right]\left[y x_{m} z\right]\left[z x_{m}\right],
$$

and therefore by a second induction on the "length" of an element in $U_{w}$, one sees that $u \in U_{(00)} \cap G^{\mathbf{b}} \Rightarrow\left[y x_{m}\right]=1,\left[z x_{m}\right]=1$. Secondly, suppose $u_{2}:=\left[x_{1}, \ldots, x_{m-1}\right] \in U_{(0)}$. Then $u=u_{2}^{-1}\left(u_{2}^{x_{m}} u^{\prime}\right) \in G^{\mathrm{b}} \Rightarrow u_{2}=1$. And argue similarly if $u=x^{-1}$.

Hence if follows that $U_{(00)} \cap G^{\mathfrak{b}}=\{1\}$, which completes the proof of the proposition.

\section{Characters of affine Kac-Moody groups}

It is the aim of this section to give the subdomain in $G$ on which a (pointwise) character of $V^{\omega}, \omega \in \mathscr{I} \mathrm{nt}_{+} \cap \mathfrak{h}_{\mathbf{Z}}^{*}$ can be defined.

3.1. Let $A$ be a type (2) affine Cartan matrix. Index the simple roots by $\{0,1, \ldots, l\}$ 
where $A_{0}$, of finite type (1), is obtained by deleting the 0 vertex in the Coxeter-Dynkin diagram of $A$. Here $\left\{h_{0}, h_{1}, \ldots, h_{l}, d\right\} \subseteq \mathfrak{h}_{\mathbf{Z}}$ where $\alpha_{i}(d)=0, i \in\{1, \ldots, l\}, \alpha_{0}(d)=1$ and rank $\mathfrak{h}_{\mathbf{z}}=l+2$ (see (1.1),(2.1)). Let the components of the least positive imaginary root $\delta \in \sum_{i=0}^{l} \mathbb{N} \alpha_{i}$ be $\delta=\left(a_{0}, a_{1}, \ldots, a_{l}\right)$. That is $\underline{a} \in \mathbb{N}^{l+1}$ is of least height and $\mathbb{R} a$ is the kernel of the quadratic form on $\mathbb{R}^{l+1}$ associated to $A$. In the dual $A^{v}=A^{t}$ write $\delta^{v}=$ $\left(a_{0}^{\vee}, a_{1}^{\vee}, \ldots, a_{l}^{\vee}\right)$, (so in each case $a_{0}^{\vee}=1$ [4]); then the affine Kac-Moody Lie algebra $\mathfrak{g}=\mathfrak{g}(A)$ has a 1 -dim centre containing the canonical central element $c=\sum_{i=0}^{l} a_{i}^{\vee} h_{i}$.

In general a real root $\alpha \in \Phi_{+}^{\text {re }}$ has $\operatorname{coroot} \alpha^{v} \in \mathbb{N} \Delta^{\vee}$ by $W$. The reflection $r_{\alpha}=w r_{i} w^{-1}$ if $w \alpha_{i}=\alpha$. For symmetrizable $A$, the $W$ invariant form (,) on $g$ (see (1.1)) is chosen such that $v\left(\Delta^{\vee}\right)=\Delta D$. And for $A$ affine take $D=\operatorname{diag}\left(a_{0} a_{0}^{\vee-1}, a_{1} a_{1}^{\vee-1}, \ldots, a_{l} a_{l}^{v-1}\right)$. Define $\Delta_{0}=\Delta \backslash\left\{\alpha_{0}\right\}, \mathfrak{h}_{0 \mathrm{Z}}=\mathfrak{h}_{\mathrm{Z}} \cap \mathbb{Q} \Delta_{0}^{\vee}, W_{0}=\left\langle r_{i} ; i \neq 0\right\rangle \leqq W, \Phi_{0}=W_{0} \Delta_{0}$ and $\mathfrak{g}_{0}=\mathrm{g}\left(A_{0}\right)$. Denote by $\theta \in \Phi_{0+}$ the highest root; then $h_{0}=c-a_{0} \theta^{v}, \delta=a_{0} \alpha_{0}+\theta$. Let $\Upsilon$ be the translation subgroup of $W$ generated by $w r_{0} r_{\theta} w^{-1}, w \in W_{0}$. Then $\Upsilon \triangleleft W$ and $W=W_{0} \propto \Upsilon$.

3.2. Lemma 2. (i) The "derivation element" $d$ acts semisimply on $V^{\omega}$ with finite dimensional eigenspaces.

(ii) The character $\delta \in \operatorname{Hom}\left(T, \mathbb{C}^{*}\right)$ extends trivially to $\delta \in \operatorname{Hom}\left(G, \mathbb{C}^{*}\right)$.

Proof. (i) If $\lambda=\omega-\sum_{i} c_{i} \alpha_{i} \in P^{\omega}$ we have $d . V_{\lambda}=\left(\omega(d)-c_{0}\right) V_{\lambda}$. The parabolic subgroup $P_{J}, J=\{1, \ldots, l\}$ of $G$ is of finite type. Thus (see (1.4)) $V_{(m)}=\sum_{\operatorname{dep}_{J}(\lambda) \leqq m}^{\oplus} V_{\lambda}$ is finite dimensional $\forall m \in \mathbb{N}$.

(ii) This is a corollary to (1.4) Proposition 1.

Let $G_{0}$ be the almost simple, complex Lie group with root datum $\left(\mathfrak{h}_{0 z}, \Delta_{0}^{\vee}, \Delta_{0}\right)$. Thus $\mathfrak{h}_{0 z}^{*}$ is the character group of $T_{0}=T \cap G_{0} \leqq G_{0}$ a maximal (algebraic) torus, and $\mathfrak{h}_{0 z} / \mathbb{Z} \Delta_{0}^{\vee}$ is the fundamental group. There is a homomorphic image of $G_{0}$ as a subgroup of $G$. Now $T=Z T_{0} T_{1}$ where $Z=\{\exp a c ; c \in \mathbb{C}\}$ is contained in the centre of $G$ and $T_{1}=\left\{\exp \left(a / a_{0}\right) d ; a \in \mathbb{C}\right\}$. Thus $\delta$ is trivial on $Z T_{0}$ and $\delta(t)=e^{\mathrm{a}}, t \in T_{1}$. Denote $T_{\mathrm{c}}=$ $T \cap K$.

Lemma 3. $K \subseteq \operatorname{Ker}|\delta|$.

Proof. This is because $K^{\prime}=\left\langle K_{i} ; i=0,1, \ldots, l\right\rangle, K_{i}=\phi_{i}(\mathrm{SU}(2))$ and $K_{i}=\bigcup_{k \in K_{i}} k_{i} T k^{-1}$ with ${ }_{i} T=T \cap K_{i} \simeq \mathrm{U}(1)$. Then $Z K^{\prime} \subseteq \operatorname{Ker} \delta$. Also $T_{\mathrm{c}} \cap T_{1}=\{\exp \sqrt{-1} \pi a d ; a \in \mathbb{R}\}$ and $G=T_{1} \ltimes G^{\prime}$.

3.3. In general the set of functions $\left\{f: \mathfrak{h}^{*} \rightarrow \mathbb{Z} ; \operatorname{supp} f \subseteq \bigcup_{j=1}^{m} \lambda_{j}-\mathbb{N} \Delta, \lambda_{j} \in \mathfrak{h}^{*}\right\}$ becomes a commutative associative algebra $E$, with unit, under convolution. Introduce $\mathrm{e}^{i} \in E, \lambda \in \mathfrak{h}^{*}$ by $\mathrm{e}^{i}(\mu)=\delta_{i \mu}$. The formal character $\chi^{\omega}$ of $V^{\omega}, \omega \in \mathscr{I} \mathrm{nt}+\cap \mathfrak{h}_{\mathcal{Z}}^{*}$ is given by $\chi^{\omega}=\sum_{i \in p_{\omega}}\left(\operatorname{dim} V_{\lambda}\right) e^{\lambda} \in E$, which can be expressed as the "Weyl-Kac" formula. The exact sequence $0 \rightarrow \mathfrak{h}_{\mathbf{z}} \stackrel{\lrcorner}{\rightarrow} \stackrel{\exp }{\rightarrow} T \rightarrow 1$ where $\imath(h)=h \otimes 1$ and $\exp (h \otimes a)=h \otimes \mathrm{e}^{2 \pi \sqrt{-1} a}, h \in \mathfrak{h}_{\mathbf{z}}, a \in \mathbb{C}$, gives to $\lambda \in \mathfrak{h}_{\mathbf{Z}}^{*}$ the character of $T, \mathrm{e}^{i}(t)=\mathrm{e}^{2 \pi \sqrt{-1} a \lambda(h)}, t=\exp (h \otimes a)$. Then, analytically, the 
region of absolute convergence of $\chi^{\omega}$ (an open, convex, $W$-invariant set in $\mathfrak{h}$ ) has been found in [4].

Returning to $A$ affine, define for a subgroup (or subset) $H$ of $G, H^{>1}=\{h \in H ;|\delta(h)|>$ 1 ) and similarly $H^{<1}, H^{=1}$. Also $H^{\neq 1}=H^{<1} \cup H^{>1}$.

Theorem 1. (i) $T^{\mathrm{tr}}=T^{>1}=\left(T^{\mathrm{hs}}\right)^{2}$,

(ii) $T^{\mathrm{b}}=Z T_{\mathrm{c}} \cup T^{>1}$.

Proof. Using the estimate multi ${ }_{\omega} \lambda \leqq K(\omega-\lambda)$ (where $K(\cdot)$ is the Kostant partition function) and mult $\alpha=1, \alpha \in \Phi^{\text {re }}$, mult $y=l, \gamma \in \Phi^{\text {im }}$ one sees that the region of absolute convergence of $\chi^{\omega}$ is given by the interior of the "Tits cone", $\{h \in \mathfrak{h} ; \operatorname{Re} \delta(h)>0\}$ where $\chi^{\omega}$ defines a holomorphic function (see [4, p. 138]).

Let $b_{\lambda}^{\omega}=\sum_{m=0}^{\infty} \operatorname{mult}_{\omega}(\lambda-m \delta) \mathrm{e}^{-\mathrm{m} \delta}$, and $W_{\lambda}$ the stabilizer of $\lambda$ in $W$. Notice that $W_{\lambda} \cap \Upsilon=\{1\}, \lambda \in P^{\omega}$. Then the formal character splits into a sum over the orbits of $\Upsilon$ on $\max (\omega)$ as

$$
\chi^{\omega}=\sum_{\lambda \in \max (\omega)} \mathrm{e}^{\lambda} b_{\lambda}^{\omega}=\sum_{\substack{\lambda \in \max (\omega) \\ \lambda \bmod Y}}\left(\sum_{\tau \in Y} \mathrm{e}^{\tau(\lambda)}\right) b_{\lambda}^{\omega} .
$$

The powers of the translation element $\tau_{v(\theta v)}=r_{\alpha_{0}} r_{\theta}$ are given by (see [4, p. 74])

$$
\begin{aligned}
\tau_{v(\theta \vee}(\lambda)= & \lambda+\lambda(c) v\left(\theta^{\vee}\right)-\left(\lambda\left(\theta^{\vee}\right)+\frac{1}{2}\left|\theta^{\vee}\right|^{2} \lambda(c)\right) \delta \\
\tau_{v(\theta \vee)}^{m}(\lambda)= & \lambda+m \lambda(c) v\left(\theta^{\vee}\right)-\left(m \lambda\left(\theta^{\vee}\right)+\frac{m}{2}\left|\theta^{\vee}\right|^{2} \lambda(c)\right. \\
& \left.+\frac{1}{2} m(m-1) \lambda(c) v\left(\theta^{\vee}\right)\left(\theta^{\vee}\right)\right) \delta, m \in \mathbb{Z}, \lambda \in \mathfrak{h}^{*} .
\end{aligned}
$$

Here $a_{0} v\left(\theta^{v}\right)=\theta$ the highest root of $\Phi_{0_{+}}$. We know $w \delta=\delta, \forall w \in W$. Also $\delta(d)=$ $a_{0}, \theta(c)=0=\theta(d)$.

Let $t \in T$ with $|\delta(t)| \leqq 1$ so $t=\exp h, \operatorname{Re} \delta(h) \leqq 0$. Consider the translations $w \tau_{v(\theta v)}^{m} w^{-1}(\lambda)$ with $w \in W_{0}$ chosen so that $w^{-1}\left(h \bmod \mathbb{C} c+\mathbb{C} d+\sqrt{-1} \mathfrak{h}_{\mathrm{OR}}\right)$ lies in the fundamental chamber for $\left(\mathfrak{g}_{0}, \mathfrak{h}_{0 z}\right)$, and $\lambda=w(\omega)$ to see that $\chi^{\omega}(t)$ diverges.

The assertions follow.

Proposition 6. (i) $B^{\text {tr }}=B^{>1} \subseteq B^{b}$,

(ii) $B^{>1}=\left(B^{\mathrm{hs}}\right)^{2}$.

Proof. (i) It is evident (since unipotent elements are upper triangular) that $b=$ $t u \in B^{\text {tr }} \Leftrightarrow t \in T^{\text {tr }}$ and $B^{\text {tr }}=T^{\text {tr }} U=T^{>1} U=B^{>1}$. The Levi subgroup $L_{\alpha}$ has, by Proposition 2, the Cartan decomposition $L_{\alpha}=K_{\alpha} T K_{\alpha}$ where $K_{\alpha}=L_{\alpha}^{\prime} \cap K \leqq L_{\alpha}^{\prime}$ is maximally compact, $\alpha \in \Phi^{\mathrm{re}}$. Then by Lemma 3 , we have $L_{\alpha}^{>1}=K_{\alpha} T^{>1} K_{\alpha} \subseteq G^{\mathrm{b}}$ from Theorem 1. An 
element $b=t u_{1} \cdots u_{m} \in B, t \in T^{>1}$, on taking " $m$ th root" $t=t_{1} \cdots t_{m}$ can be written $b=t_{1} u_{1}^{\prime} \cdots t_{m} u_{m}^{\prime}$ with each $t_{j} u_{j}^{\prime} \in L_{\beta_{j}}^{>1}, \beta_{j} \in \Phi_{+}^{\mathrm{re}}, j \in\{1, \ldots, m\}$. Hence $B^{>1} \subseteq B^{\mathrm{b}}$.

(ii) Follows from (i) and Theorem 1 as

$$
L_{a}^{>1} \cap B=T^{>1}\left(L_{a}^{>1} \cap B\right) \subseteq T^{>1} B^{\mathrm{hs}} \subseteq\left(B^{\mathrm{hs}}\right)^{2}
$$

Also $\left(B^{\text {hs }}\right)^{2} \subseteq B^{\text {tr }}$

Lemma 4. $\left(G^{\mathrm{cl}}\right)^{-1}=G^{\mathrm{cl}}$.

Proof. We know that $T / T_{\mathrm{c}} \cap T_{1} \subseteq G^{\text {sym }} \subseteq G^{\mathrm{cl}}$. Also given any $g \in G$, using (1.4) and Proposition 6(i), $\exists t \in T / T_{\mathrm{c}} \cap T_{1}^{>1}$ with $\mathrm{R}\left(\mathrm{tg}^{-1}\right)$ bounded.

Let $\left(x_{n}\right)$ be a convergent sequence in $V^{\omega}$ with $\mathbf{R}(g) x_{n} \rightarrow 0$. Then $\mathbf{R}(t) x_{n}=$ $\mathbf{R}\left(\operatorname{tg}^{-1}\right) \mathbf{R}(g) x_{n} \rightarrow 0$. But $\mathbf{R}\left(t^{-1}\right)$ is closeable, thus $x_{n} \rightarrow 0$.

Hence we have shown that if $g \in G^{\text {el }}$ then $\overline{R(g)}$ is injective on dom $\overline{R(g)}$, which gives the lemma.

Corollary. $G^{\mathrm{cl}}=G$.

Proof. We know that $G^{\mathrm{cl}} G^{\mathrm{b}} \subseteq G^{\mathrm{cl}}$ and $G^{\mathrm{b}} \subseteq G^{\mathrm{cl}}$.

Let $g \in G$. So as above $\exists t \in T^{>1}$ with $t g^{-1} \in G^{\mathrm{b}}$. Therefore $g t^{-1} \in G^{\mathrm{cl}}$ giving $g=$ $\left(g t^{-1}\right) t \in G^{\mathrm{cl}}$.

Proposition 7. (i) $B^{<1}=T^{<1} U \subseteq G \backslash G^{\text {b }}$,

(ii) $T^{=1}(U \backslash\{1\}) \subseteq G \backslash G^{\mathbf{b}}$.

Proof. One has $T^{=1}=Z T_{\mathrm{c}} T_{0}^{\text {sym }}$. Taking into account (2.3) Proposition 5 and Theorem 1 (ii) in (3.3), we want to show that $t_{0} u \in G \backslash G_{\mathrm{b}}$ with $t_{0} \in T_{0}^{\text {sym }}, t_{0} \neq 1, u \in U \backslash\{1\}$.

The formula in (3.3) for the power of an element in $\Upsilon$ and the character formula $\chi^{\omega}$ give that for $\lambda \in \max (\omega)$, taking a conjugate $\mu=w \tau_{v\left(\theta^{v}, w^{-1}\right.}(\lambda), w \in W_{0}, t_{0}=\exp h$ and $w^{-1}(h)$ in the fundamental chamber of $\left(\mathfrak{g}_{0}, \mathfrak{h}_{0 z}\right)$, we have

$$
\left\langle R\left(t_{0} u\right) z, z\right\rangle=\left\langle\mathrm{R}\left(t_{0}\right) z, z\right\rangle=\mathrm{e}^{\lambda(h)+m \omega(c) \theta\left(w^{-1} h\right) / a_{0}}
$$

where $z$ has weight $\mu,\|z\|=1$.

Theorem 2. (0) $G^{\mathrm{b}}=K B^{\mathrm{b}}, B^{\mathrm{b}}=B^{>1} \cup\left(B^{=1} \cap T^{\mathrm{b}}\right)$,

(1) $G^{\mathrm{b}} \cap G^{\mathrm{tr}} \supseteq G^{>1}=\left(G^{\mathrm{hs}}\right)^{2}=G^{\mathrm{hs}}$,

(2) $G^{\mathrm{cpt}}=G^{\mathrm{hs}}$.

Proof. (0) We have $G=K B, K G^{\mathrm{b}}=G^{\mathrm{b}}, B^{>1} \subseteq B^{\mathrm{b}}, B^{<1} \cap B^{\mathrm{b}}=\emptyset$. Also $B^{=1} \cap B^{\mathrm{b}}=$ $Z T_{\mathrm{c}}=T^{=1} \cap T^{\mathrm{b}}$. 
(1) Follows from (3.2) Lemma 3 and (3.3) Proposition 6.

(2) From (1) and (3.3) Theorem $1, G^{\mathrm{cpt}}=K B^{\mathrm{cpt}}=K B^{>1}=G^{>1}=G^{\mathrm{hs}}$.

3.4. Conjugation invariance. Let $G$ be of type (1), (2) or (3). Take $G(\emptyset)$ the union of the Borel subgroups of $G$; that is the set of elements of $G$ which are conjugate under $G$ $(\Rightarrow$ under $K)$ into the standard Borel subgroup $B$.

Proposition 8. Let $x \in\left(G^{\mathrm{hs}}\right)^{2} \cap G(\emptyset)$, and $g \in G$ with $g x g^{-1} \in\left(G^{\mathrm{hs}}\right)^{2}$, then

$$
\operatorname{trace}_{\omega} \mathbf{R}\left(g x g^{-1}\right)=\operatorname{trace}_{\omega} \mathrm{R}(x), \forall \omega \in \mathscr{I} \mathrm{nt}_{+} \cap \mathfrak{h}_{\mathbf{Z}}^{*} \text {. }
$$

Proof. By definition $\exists k_{1} \in K$ with $k_{1}^{-1} x k_{1}=b \in B$. Also $\exists k \in K, b_{1} \in B$ with $g k_{1}=k b_{1}$ giving $g x g^{-1}=k b_{1} b b_{1}^{-1} k^{-1}$. Then from (2.2), $\forall \omega \in \mathscr{I} \mathrm{nt}+\cap \mathfrak{h}_{\mathbf{Z}}^{*}$,

$$
\operatorname{trace}_{\omega} \mathbf{R}\left(g x g^{-1}\right)=\operatorname{trace}_{\omega} \mathbf{R}\left(b_{1} b b_{1}^{-1}\right)=\operatorname{trace}_{\omega} \mathbf{R}(b)=\operatorname{trace}_{\omega} \mathbf{R}(x) .
$$

\section{Lemma 5.}

$$
\operatorname{trace}_{\omega} \mathbf{R}\left(\operatorname{tgt}^{-1}\right)=\operatorname{trace}_{\omega} \mathrm{R}(g), \forall g \in G, \forall t \in T, \forall \omega \in \mathscr{I} \mathrm{nt}_{+} \cap \mathfrak{h}_{\mathbf{Z}}^{*}
$$

Proof. In fact writing $t=t_{1} t_{2}, t_{1} \in T \cap K, t_{2} \in T^{\text {sym }}$ (the polar decomposition), a matrix element

$$
\begin{aligned}
\left\langle R\left(g t^{-1}\right) z, z\right\rangle & =\mathrm{e}^{\lambda}\left(t^{-1}\right)\left\langle\mathrm{R}(g) z, \mathrm{R}\left(t_{1}^{-1} t_{2}\right) z\right\rangle \\
& =\mathrm{e}^{\lambda}\left(t^{-1}\right) \overline{\mathrm{e}^{\lambda}\left(t_{1}^{-1}\right)} \mathrm{e}^{\lambda}\left(t_{2}\right)\langle\mathrm{R}(g) z, z\rangle=\langle\mathrm{R}(g) z, z\rangle
\end{aligned}
$$

where $z$ is of weight $\lambda$.

Now let $G$ be of type (2).

Theorem 3.

$$
\operatorname{trace}_{\omega} \mathrm{R}\left(g x g^{-1}\right)=\operatorname{trace}_{\omega} \mathrm{R}(x), \forall x \in G^{>1}, \forall g \in G
$$

Proof. With $g=k b, k \in K, b \in B, b=u \bmod T, x=x_{1} x_{2}, x_{1}, x_{2} \in G^{>1}$ we have from (2.2), Lemma 5 and Theorem 2 that

$$
\begin{aligned}
\operatorname{trace}_{\omega} \mathrm{R}\left(g x g^{-1}\right) & =\operatorname{trace}_{\omega} \mathrm{R}\left(u x u^{-1}\right)=\operatorname{trace}_{\omega} \mathrm{R}\left(\left(u x_{1}\right)\left(x_{2} u^{-1}\right)\right) \\
& =\operatorname{trace}_{\omega} \mathrm{R}\left(x_{2} x_{1}\right)=\operatorname{trace}_{\omega} \mathrm{R}(x) .
\end{aligned}
$$

Acknowledgements. This work was carried out while at the University of Liverpool 
1987-1989. My thanks to Peter Slodowy for making helpful comments and suggestions. Also thanks to the referee for comments. I acknowledge the support of the U.K. SERC.

\section{REFERENCES}

1. R. W. CARTER, Finite Groups of Lie Type: Conjugacy Classes and Complex Characters (J. Wiley, 1985).

2. A. J. Feingold and J. LepowsKY, The Weyl-Kac character formula and power series identities, Adv. Math. 29 (1978), 271-309.

3. H. Garland, The arithmetic theory of loop algebras, Publ. Math. I.H.E.S. 52 (1980), 5-36.

4. V. G. KAC, Infinite Dimensional Lie Algebras (2nd edition, C.U.P. (1985)).

5. V. G. KAC, Constructing groups associated to infinite dimensional Lie algebras, in Infinite Dimensional Groups with Applications (ed. V. G. Kac, MSRI Publ. 4, Springer-Verlag, 1985).

6. V. G. KAC and D. H. Peterson, Unitary structure in representations of infinite dimensional groups and a convexity theorem, Invent. Math. 76 (1984), 1-14.

7. E. LoOJJenGa, Invariant theory for generalized root systems, Invent, Math. 61 (1980), 1-32.

8. A. Pressley and G. Segal, Loop Groups (O.U.P. 1988).

9. P. SLodowy, An adjoint quotient for certain groups attached to Kac-Moody algebras Infinite Dimensional Groups with Applications (ed. V. G. Kac, MSRI Publ. 4, Springer-Verlag, 1985).

10. P. Stodowy, A character approach to Looijenga's invariant theory for generalized root systems, Compositio Math. 55 (1985), 3-32.

11. P. Stodowy, On the Geometry of Schubert Varieties Attached to Kac-Moody Lie Algebras, (Canadian Math. Soc. Proceedings 6, 1986).

12. J. TITs, Uniqueness and presentation of Kac-Moody groups over fields, J. Algebra 105 (1987), 542-573.

13. N. R. Wallach, Real Reductive Groups I (Academic Press, 1988).

14. J. Weidmann, Linear Operators in Hilbert Spaces (GTM, Springer-Verlag, 1980).

Department of Pure Mathematics

UNIVERSITY OF LIVERPOOL

P.O. Box 147

LIVERPOOL L69 3BX
Present address:

Department of Pure Mathematics UNIVERSITY OF LEEDS

LEEDS LS2 9JT 